PROCEEDINGS OF THE

AMERICAN MATHEMATICAL SOCIETY

Volume 131, Number 8, Pages 2423-2433

S 0002-9939(02)06764-3

Article electronically published on November 13, 2002

\title{
LOCAL RADIAL PHRAGMÉN-LINDELÖF ESTIMATES FOR PLURISUBHARMONIC FUNCTIONS ON ANALYTIC VARIETIES
}

\author{
RÜDIGER W. BRAUN, REINHOLD MEISE, AND B. A. TAYLOR
}

(Communicated by Juha M. Heinonen)

\begin{abstract}
We give a sufficient condition for a local radial Phragmén-Lindelöf principle on analytic varieties. This condition is expressed in terms of existence of hyperbolic directions.
\end{abstract}

Introduction 1. In a basic paper Hörmander 12 characterized when a given linear partial differential operator $P(D)$ with constant coefficients is surjective on the space $\mathcal{A}(\Omega)$ of all real-analytic functions on an open convex subset $\Omega$ of $\mathbb{R}^{n}$. His characterization was given in terms of global and also of local conditions of Phragmén-Lindelöf type for plurisubharmonic functions on the zero variety of the symbol $P$. Since then, it was shown in a number of papers that similar PhragménLindelöf conditions on algebraic varieties can be used to characterize other properties of (systems of) such operators (see, e.g., Andreotti and Nacinovich [1], Boiti and Nacinovich [3], Braun, Meise, and Vogt [9], Franken and Meise [11], Kaneko [13], Meise, Taylor, and Vogt [15], Momm [18], Palamodov [20, Zampieri [24]).

This work motivates the challenging complex analysis problem of characterizing geometrically the varieties for which such Phragmén-Lindelöf estimates are valid. The present authors, along with D. Vogt, have studied this question in [4, 5], 6], [8], [9], [14, [15, [16, 17]. The main result of this paper, Theorem 10, gives a local geometric condition on an analytic variety near a real point $\xi$ which guarantees that any plurisubharmonic function $u$ on the variety that vanishes on its real points can grow only linearly, $u(z)=O(|z-\xi|)$, near $\xi$. The geometric condition, which is described in Definition 9 is expressed in terms of hyperbolicity and is the local analog of a global version given in [5]. Unfortunately, the condition is not necessary, as we will show in Example 14. As in the global case, the proof of the theorem is based on a result of Sibony-Wong type for homogeneous algebraic varieties. We were led to Theorem 10 because it is a key result from pluripotential theory that is needed in our recent characterization in [8] of those surfaces in $\mathbb{C}^{3}$ that satisfy the local Phragmén-Lindelöf condition. This characterization is applied in 8 to extend Hörmander's characterization of the surjective $P(D)$ on $\mathcal{A}\left(\mathbb{R}^{n}\right)$ from $n=3$ to $n=4$.

Received by the editors October 2, 2000 and, in revised form, March 11, 2002.

2000 Mathematics Subject Classification. Primary 32U05, 32U15.

The authors gratefully acknowledge support of DAAD under the program "Projektbezogene Förderung des Wissenschaftleraustauschs mit den USA in Zusammenarbeit mit der National Science Foundation". 
To formulate the results clearly, we need some preparation:

Notation 2. Throughout the paper, $|\cdot|$ denotes the Euclidean norm on $\mathbb{C}^{n}$. For $\xi \in \mathbb{C}^{n}$ and $r>0$ we let

$$
B(\xi, r):=\left\{z \in \mathbb{C}^{n}:|z-\xi|<r\right\}
$$

and for $\xi \in \mathbb{R}^{n},|\xi|=1, \epsilon>0$, and a zero neighborhood $D \subset B(0,1)$ we define the truncated cone $\Gamma(\xi, D, \epsilon)$ with profile $D$ by

$$
\Gamma(\xi, D, \epsilon):=\bigcup_{0<t<\epsilon} t(\xi+D) .
$$

Definition 3. (a) An analytic variety $V$ in an open set $G$ in $\mathbb{C}^{n}$ is defined to be a closed analytic subset of $G$ (see Chirka [10, 2.1).

(b) Let $V$ be an analytic variety in some open set in $\mathbb{C}^{n}$ and let $\Omega$ be an open subset of $V$. A function $u: \Omega \rightarrow[-\infty, \infty$ [ is called plurisubharmonic if it is locally bounded above, plurisubharmonic in the usual sense on $\Omega_{\text {reg }}$, the set of all regular points of $V$ in $\Omega$, and satisfies

$$
u(z)=\limsup _{\zeta \in \Omega_{\mathrm{reg}}, \zeta \rightarrow z} u(\zeta)
$$

at the singular points of $V$ in $\Omega$. By $\operatorname{PSH}(\Omega)$ we denote the set of all plurisubharmonic functions on $\Omega$.

It is easy to check that the following definition is equivalent to the one given in Meise, Taylor, and Vogt [16], 2.3 (see Lemma 7 below).

Definition 4. Let $V$ be an analytic variety in some ball $B(\xi, r)$ for $\xi \in V \cap \mathbb{R}^{n}$ and $r>0$. We say that $V$ satisfies the condition $\operatorname{RPL}_{\text {loc }}(\xi)$ if the following holds:

There exist $A>0$ and $0<r_{2} \leq r_{1} \leq r$ such that each plurisubharmonic function $u$ on $V \cap B\left(\xi, r_{1}\right)$ which satisfies

( $\alpha) u(z) \leq 1, \quad z \in V \cap B\left(\xi, r_{1}\right)$, and

(ß) $u(z) \leq 0, \quad z \in V \cap B\left(\xi, r_{2}\right) \cap \mathbb{R}^{n}$,

already satisfies

$(\gamma) u(z) \leq A|z-\xi|, \quad z \in V \cap B\left(\xi, r_{1}\right)$.

Definition 5. Let $V \subset \mathbb{C}^{n}$ be an analytic variety in some ball $B(p, r), p \in \mathbb{C}^{n}, r>$ 0 . Let $T_{p} V$ denote the tangent cone to $V$ at $p$ in the sense of Whitney 23, 7.1G. To describe $T_{p} V$ in an equivalent way, let $f$ be analytic in some neighborhood of a point $p$. Then the localization $f_{p}$ of $f$ at the point $p$ is defined as the lowest degree homogeneous polynomial in the Taylor series expansion of $f$ at $p$ which does not vanish. With this notation we have

$T_{p} V=\left\{z \in \mathbb{C}^{n}: f_{p}(z)=0\right.$ for all $f$, analytic near $p$ and vanishing on $\left.V\right\}$,

by Whitney 23, 7.4D.

Proposition 6. Let $V$ be an analytic variety in some open set in $\mathbb{C}^{n}$. If $V$ satisfies $\mathrm{RPL}_{\mathrm{loc}}(\xi)$ for some $\xi \in V \cap \mathbb{R}^{n}$, then $T_{\xi} V$ satisfies $\mathrm{RPL}_{\mathrm{loc}}(0)$.

Proof. It is no restriction to assume $\xi=0$. We may also assume that $V$ is a subvariety of $B(0,1)$ and that $\mathrm{RPL}_{\mathrm{loc}}(0)$ holds for the parameters $A>0,0<r \leq$ $1=r_{1}$. Then define the following varieties $V_{j}$ in $B(0,1)$ for $j \in \mathbb{N}_{0}$ :

$$
V_{0}:=T_{0} V \cap B(0,1), V_{j}:=\{z \in B(0,1): z / j \in V\}=j(V \cap B(0,1 / j)), j \in \mathbb{N} .
$$


Furthermore, define, for $j \in \mathbb{N}_{0}$, the extremal functions $U_{j}: V_{j} \rightarrow \mathbb{R}$ as in Meise, Taylor, and Vogt [16], 4.1:

$$
\begin{aligned}
& U_{j}(z):=\sup \left\{u(z): u \in \operatorname{PSH}\left(V_{j}\right), u(z) \leq 1 \text { for } z \in V_{j},\right. \\
& \left.\qquad u(z) \leq 0 \text { for } z \in V_{j} \cap \mathbb{R}^{n} \cap \overline{B(0,1 / 2)}\right\} .
\end{aligned}
$$

Then $\lim _{j \rightarrow \infty} V_{j}=T_{0} V$, either as currents on $\mathbb{C}^{n}$ (see Chirka [10], 16.1, Proposition 2) or in the sense of Meise, Taylor, and Vogt [16], 4.3. By 4.4 of [16], if $z_{j} \in V_{j}$ and $\lim _{j \rightarrow \infty} z_{j}=: z \in V_{0}$, then

$$
U_{0}(z) \leq \liminf _{j \rightarrow \infty} U_{j}\left(z_{j}\right) .
$$

Consequently, the proposition is proved once we show

(*) There exists $B>0$ such that $U_{j}(z) \leq B|z|$ for each $j \in \mathbb{N}$ and each $z \in V_{j}$.

To prove $(*)$ we define (as in [16], 2.9)

$$
H: \mathbb{C}^{n} \rightarrow \mathbb{R}, \quad H(z)=\frac{1}{2}\left(|\operatorname{Im} z|^{2}-|\operatorname{Re} z|^{2}\right),
$$

and note that $H$ is pluriharmonic on $\mathbb{C}^{n}$. Next fix $j \in \mathbb{N}$ and $u \in \operatorname{PSH}\left(V_{j}\right)$ satisfying

$$
u(z) \leq 1 \text { for } z \in V_{j} \text { and } u(z) \leq 0 \text { for } z \in V_{j} \cap \mathbb{R}^{n} \cap B(0,1 / 2)
$$

and define $w: V \rightarrow \mathbb{R}$ by

$$
w(z):= \begin{cases}\max \left(\frac{1}{j} u(j z)+\frac{3}{j} H(j z), 3|\operatorname{Im} z|\right), & \text { if }|z|<\frac{1}{2 j} \\ 3|\operatorname{Im} z|, & \text { if }|z| \geq \frac{1}{2 j}\end{cases}
$$

For $z \in V$ with $|z|=\frac{1}{2 j}$ we have

$$
\frac{1}{j} u(j z)+\frac{3}{j} H(j z) \leq \frac{1}{j}+\frac{3}{j}\left(|\operatorname{Im} j z|-\frac{1}{2}\right) \leq 3|\operatorname{Im} z|-\frac{1}{2 j} .
$$

This implies $w \in \operatorname{PSH}(V)$. Moreover, the maximum principle gives $w(z) \leq 3$ for $z \in V$. The hypotheses on $u$, the definition of $w$ and $H \mid \mathbb{R}^{n} \leq 0$ imply $w(z) \leq 0$ for $z \in V \cap \mathbb{R}^{n}$. Since $V$ satisfies $\mathrm{RPL}_{\mathrm{loc}}(0)$ for the parameters $A>0$ and $0<r \leq 1$, we obtain

$$
w(z) \leq 3 A|z|, \quad z \in V .
$$

By the definition of $w$ and the definition of $H$ we now get for $z \in V,|z|<\frac{1}{2 j}$ :

$$
3 A|z| \geq w(z) \geq \frac{1}{j} u(j z)+\frac{3}{j} H(j z) \geq \frac{1}{j} u(j z)-\frac{3}{2 j}|j z|^{2} .
$$

This implies

and hence

$$
u(j z) \leq\left(3 A+\frac{3}{2}|j z|\right)|j z| \leq\left(3 A+\frac{3}{4}\right)|j z|
$$

$$
u(\zeta) \leq 3(A+1)|\zeta|, \quad \zeta \in V_{j} \cap B(0,1 / 2) .
$$

Moreover, for $\zeta \in V_{j}$ satisfying $1 / 2 \leq|\zeta|<1$ the hypotheses on $u$ imply $u(\zeta) \leq$ $1 \leq 2|\zeta|$. Hence (11) holds on $V_{j}$. Condition $(*)$ follows because $U_{j}(z)$ is the upper envelope of all such $u(z)$. This completes the proof. 
Using localization arguments as in the proof of Proposition 6, one can show that the particular choice of $r_{1}$ and $r_{2}$ in the definition of $\mathrm{RPL}_{\mathrm{loc}}$ does not matter.

Lemma 7. Let $V$ be an analytic variety in $B(\xi, r)$ for some $r>0$ and $\xi \in V \cap \mathbb{R}^{n}$. Then $V$ satisfies $\mathrm{RPL}_{\mathrm{loc}}(\xi)$ if and only if the following condition is satisfied:

For all choices $0<r_{2} \leq r_{1} \leq r$ there exists $A>0$ such that each $u \in$ $\operatorname{PSH}\left(V \cap B\left(\xi, r_{1}\right)\right)$ which satisfies

( $\alpha) u(z) \leq 1$ for $z \in V \cap B\left(\xi, r_{1}\right)$,

(B) $u(z) \leq 0$ for $z \in V \cap \mathbb{R}^{n} \cap B\left(\xi, r_{2}\right)$,

also satisfies

$(\gamma) u(z) \leq A|z-\xi|$ for $z \in V \cap B\left(\xi, r_{1}\right)$.

The converse implication in Proposition[6] does not hold, as the following example shows.

Example 8. The variety $V:=\left\{(x, y) \in \mathbb{C}^{2}: x+i y^{2}=0\right\}$ does not satisfy $\mathrm{RPL}_{\text {loc }}(0)$, although $T_{0} V$ satisfies $\mathrm{RPL}_{\mathrm{loc}}(0)$. The last statement follows easily from a classical result (see Nevanlinna [19], 38) since $T_{0} V=\left\{(x, y) \in \mathbb{C}^{2}: x=0\right\}$.

To see that $V$ does not satisfy $\mathrm{RPL}_{\text {loc }}(0)$, note that by Meise, Taylor, and Vogt [16], 2.8, a necessary condition for $V$ to satisfy $\mathrm{RPL}_{\mathrm{loc}}(0)$ is that $V$ satisfies the dimension condition at 0 . By [16, 2.6, this means that $V \cap \mathbb{R}^{2}$ has to have real dimension 1 . Since $V \cap \mathbb{R}^{2}=\{0\}$, this is not the case.

Because of Proposition 6 and Example 8 it appears interesting to know conditions which imply that a given variety $V$ inherits $\mathrm{RPL}_{\text {loc }}$ at $\xi \in V \cap \mathbb{R}^{n}$ from its tangent cone $T_{\xi} V$. As we indicated in the Introduction, we also need to know such a condition in our paper [8]. To present a sufficient condition which covers the intended applications, we have to introduce some more notation.

Definition 9. (a) Let $V$ be an analytic variety in $\mathbb{C}^{n}$ of pure dimension $k \geq 1, p \in$ $V$ and $\pi: \mathbb{C}^{n} \rightarrow \mathbb{C}^{n}$ a projection map. We say that $\pi$ is a noncharacteristic projection for $V$ at $p$ if im $\pi$ and ker $\pi$ are spanned by real vectors, rank $\pi=k$, and $T_{p} V \cap \operatorname{ker} \pi=\{0\}$.

(b) Let $V$ be an analytic variety in some open set in $\mathbb{C}^{n}$ and $p \in V \cap \mathbb{R}^{n}$. $V$ is said to be 1-hyperbolic at $p$ with respect to $\xi \in T_{p} V \cap \mathbb{R}^{n}, \xi \neq 0$, if there exist a cone $\Gamma=\Gamma(\xi, D, \epsilon)$ and a noncharacteristic projection for $V$ at $p$ such that $\pi:(V-p) \cap \Gamma \rightarrow \pi((V-p) \cap \Gamma)$ is proper and $z \in(V-p) \cap \Gamma$ is real whenever $\pi(z)$ is real.

The expression "1-hyperbolicity" stems from our paper [8], where the more general concept of " $d$-hyperbolicity" is used.

Now we can formulate the main result of the present paper:

Theorem 10. Let $V$ be an analytic variety of pure dimension $k \geq 1$ in some ball $B(\xi, r)$ in $\mathbb{C}^{n}$, where $\xi \in V \cap \mathbb{R}^{n}$. Assume that for each irreducible component $W$ of $T_{\xi} V$ there is $\eta \in W \cap \mathbb{R}^{n}$ such that $V$ is 1-hyperbolic at $\xi$ with respect to $\eta$ and to $-\eta$. Then $V$ satisfies $\mathrm{RPL}_{\text {loc }}(\xi)$.

The proof of Theorem 10 is based on a result of Sibony-Wong type (compare Sibony and Wong [21] and Siciak [22] for the original result) which we recall from [5], 3.1, for the convenience of the reader. 
Theorem 11. Let $W$ be a homogeneous algebraic variety of pure dimension $k$ in $\mathbb{C}^{n}$ and let $\pi: W \rightarrow \mathbb{C}^{k}, \pi\left(z^{\prime}, z^{\prime \prime}\right)=z^{\prime}$, be proper. Let $\Gamma^{\prime} \subset \mathbb{C}^{k}$ be an open complex cone, let $\Gamma:=\pi^{-1}\left(\Gamma^{\prime}\right)$, and let $R \subset \Gamma$ be a complex cone which is nonpluripolar in $\Gamma$. Then there exists a constant $A \geq 1$ such that for each $u \in \operatorname{PSH}(\Gamma)$ which is positively homogeneous and satisfies

(a) $u(z) \leq|z|$ for $z \in R$,

(b) $v\left(z^{\prime}\right):=\max \left\{u\left(z^{\prime}, z^{\prime \prime}\right):\left(z^{\prime}, z^{\prime \prime}\right) \in \Gamma\right\}$ for $z^{\prime} \in \Gamma^{\prime}$, extends to a plurisubharmonic function $\tilde{v}$ on $\mathbb{C}^{k}$,

each extension $\tilde{v}$ satisfies

(c) $\tilde{v}\left(z^{\prime}\right) \leq A\left|z^{\prime}\right|, z^{\prime} \in \mathbb{C}^{k}$.

In particular, $u$ satisfies $u(z) \leq A|z|$ for all $z \in W$.

The following geometric preparation for the proof of Theorem 10 might also be of some interest by itself. While in Definition 9 it appears that 1-hyperbolicity depends on a special projection direction, it in fact does not - there are always nearby points at which $V$ is 1-hyperbolic with respect to every real noncharacteristic projection.

Proposition 12. Let $V$ be a purely $k$-dimensional analytic variety in some open neighborhood of the origin in $\mathbb{C}^{n}$, and let $\xi_{0} \in T_{0} V \cap \mathbb{R}^{n}$ with $\left|\xi_{0}\right|=1$ be given. If $V$ is 1-hyperbolic at the origin with respect to $\xi_{0}$ and to $-\xi_{0}$ and $U$ is an arbitrary neighborhood of $\xi_{0}$, then there is $\xi \in T_{0} V \cap U \cap \mathbb{R}^{n}$ which is a regular point of $T_{0} V$ and has the following property:

For each projection $\pi$ which is noncharacteristic for $V$ at 0 and which satisfies $T_{\xi}\left(T_{0} V\right) \cap \operatorname{ker} \pi=\{0\}$ there are $\epsilon>0$ and a profile $C$ such that for $\sigma= \pm 1$ the cone $\Gamma:=\Gamma(\sigma \xi, C, \epsilon)$ is such that $\pi: V \cap \Gamma \rightarrow \pi(V \cap \Gamma)$ is proper and $z \in V \cap \Gamma$ is real whenever $\pi(z)$ is real.

Proof. Since $V$ is 1 -hyperbolic at 0 with respect to $\xi_{0}$ and to $-\xi_{0}$, there are a noncharacteristic projection $\pi_{0}$, a constant $\epsilon_{0}>0$, and a profile $C_{0}$ such that, for the cones $\Gamma_{0}^{ \pm}:=\Gamma\left( \pm \xi_{0}, C_{0}, \epsilon_{0}\right)$, the projection $\pi_{0}: V \cap \Gamma_{0}^{ \pm} \rightarrow \pi_{0}\left(V \cap \Gamma_{0}^{ \pm}\right)$is proper and $z \in V \cap \Gamma_{0}^{ \pm}$is real whenever $\pi_{0}(z)$ is real. Since $\pi_{0}$ is noncharacteristic, $\xi_{0} \notin \operatorname{ker} \pi_{0}$. We may assume $\xi_{0}=(0, \ldots, 0,1)$.

Since $\pi_{0}$ is noncharacteristic, it induces a description of $T_{0} V$ as analytic cover above a neighborhood of 0 in im $\pi_{0}$. Since branching occurs only over a proper analytic subset, there is $\xi \in T_{0} V \cap U \cap \mathbb{R}^{n}$ such that $\pi_{0}: T_{0} V \rightarrow \operatorname{im} \pi_{0}$ is unbranched in a suitable neighborhood of $\xi$, in particular, $\xi$ is a regular point of $T_{0} V$. Then there are a cone $\Gamma_{1}=\Gamma\left(\xi, C_{1}, \epsilon_{1}\right) \subset \Gamma_{0}^{+}$and a holomorphic map $g: \pi_{0}\left(\Gamma_{1}\right) \rightarrow \operatorname{ker} \pi_{0}$ such that

$$
T_{0} V \cap \Gamma_{1}=\left\{v+g(v): v \in \pi_{0}\left(\Gamma_{1}\right)\right\} .
$$

Let $C_{2} \subset C_{1}$ be relatively compact, and set $\Gamma_{2}:=\Gamma\left(\xi, C_{2}, \epsilon_{1}\right)$. We claim that $w \in T_{0} V \cap \Gamma_{2}$ is real whenever $\pi_{0}(w)$ is real. To see this, fix $w \in T_{0} V \cap \Gamma_{2}$ with $\pi_{0}(w)$ real. Near the origin, the projection $\pi_{0}$ induces a description of $V$ as an analytic cover above some neighborhood of 0 in im $\pi_{0}$. Since $T_{0} V$ is the tangent cone, at least one sheet of $V$ must eventually come close to $T_{0} V \cap \Gamma_{2}$. Hence, for sufficiently large $j \in \mathbb{N}$, there is $z_{j} \in V \cap \Gamma_{2}$ with $\pi_{0}\left(z_{j}\right)=\pi_{0}(w) / j$. Then $z_{j} \in \mathbb{R}^{n}$ because $\Gamma_{2} \subset \Gamma_{0}^{+}$. Note that there is $\eta>0$ such that

$$
\eta\left|\pi_{0}(z)\right| \leq|z| \leq \frac{1}{\eta}\left|\pi_{0}(z)\right| \quad \text { for all } z \in \Gamma_{0}^{+} .
$$


Hence the points $j z_{j}$ are all in $\Gamma_{2}$ and satisfy a common upper as well as a common lower bound, where the latter is strictly positive. By compactness, this means that the sequence $\left(j z_{j}\right)_{j \in \mathbb{N}}$ has a subsequence which converges to some $w_{1} \in \bar{\Gamma}_{2} \backslash\{0\} \subset$ $\Gamma_{1}$. By definition, we have $w_{1} \in T_{0} V$ and $\pi_{0}\left(w_{1}\right)=\pi_{0}(w)$. Since $T_{0} V \cap \Gamma_{1}$ is a graph by (2), this shows $w=w_{1} \in \mathbb{R}^{n}$.

Fix now $\pi$ as in the hypothesis, then $T_{\xi}\left(T_{0} V\right)$ is a tangent hyperplane which has trivial intersection with $\operatorname{ker} \pi$. This implies that near $\xi, T_{0} V$ is a graph over $\operatorname{im} \pi$, i.e., there are a cone $\Gamma_{3}=\Gamma\left(\xi, C_{3}, \epsilon_{3}\right)$ with $0 \in \bar{C}_{3} \subset C_{2}$ and a holomorphic map $h: \pi\left(\Gamma_{3}\right) \rightarrow \operatorname{ker} \pi$ such that

$$
T_{0} V \cap \Gamma_{3}=\left\{v+h(v): v \in \pi\left(\Gamma_{3}\right)\right\}
$$

It is easy to see that, since $g$ is real valued for real arguments, so is $h$. Fix a cone $\Gamma_{4}=\Gamma\left(\pi_{0}(\xi), C_{4}, \epsilon_{4}\right) \subset \pi_{0}\left(\Gamma_{2}\right)$ such that $z \in \Gamma_{3}$ whenever $z \in V \cap \Gamma_{2}$ satisfies $\pi_{0}(z) \in \Gamma_{4}$. Let $C_{5}$ be a neighborhood of 0 , relatively compact in $C_{4}$, and set $\Gamma_{5}=\Gamma\left(\pi_{0}(\xi), C_{5}, \epsilon_{4} / 2\right)$. It follows from classical estimates of potential theory (e.g., Nevanlinna [19], 38) that there is a constant $A>0$ such that the following holds:

Whenever $u: \Gamma_{4} \rightarrow[-\infty, \infty[$ is plurisubharmonic and satisfies $u(\zeta) \leq|\zeta|$ for each $\zeta \in \Gamma_{4}$ and $u(\zeta) \leq 0$ for each $\zeta \in \Gamma_{4} \cap \mathbb{R}^{n}$, then $u(\zeta) \leq A|\operatorname{Im} \zeta|$ for each $\zeta \in \Gamma_{5}$.

Next note that we can find $\delta>0$ such that

$$
\left|\operatorname{Im} \pi_{0}(z)\right|=\left|\pi_{0}(\operatorname{Im} z)\right| \leq \frac{1}{\delta}|\operatorname{Im} z| \quad \text { for all } z \in \mathbb{C}^{n}
$$

and define $u: \Gamma_{4} \rightarrow[-\infty, \infty[$ by

$$
u(\zeta):=(A / \delta+1) \max \left\{|\operatorname{Im}(z-\pi(z)-h(\pi(z)))|: z \in V \cap \Gamma_{3}, \pi_{0}(z)=\zeta\right\} .
$$

Note that $|z-\pi(z)-h(\pi(z))|=o(|z|)$ for $z \in V \cap \Gamma_{3}$, hence $u(\zeta) \leq|\zeta|$ if we assume that $\epsilon_{3}$ is sufficiently small. If $\zeta$ is real, then so is each $z \in V \cap \Gamma_{3}$ with $\pi_{0}(z)=\zeta$, hence $u(\zeta) \leq 0$ in that case. By the above, we find $u(\zeta) \leq A|\operatorname{Im} \zeta|$ for $\zeta \in \Gamma_{5}$. Moreover, if $z \in V \cap \Gamma_{3}, \pi(z)$ is real, and $\pi_{0}(z) \in \Gamma_{5}$, then

$$
\begin{aligned}
A\left|\operatorname{Im} \pi_{0}(z)\right| \geq u\left(\pi_{0}(z)\right) \geq(A / \delta+1)|\operatorname{Im} z| & \\
& \geq \delta(A / \delta+1)\left|\operatorname{Im} \pi_{0}(z)\right|=(A+\delta)\left|\operatorname{Im} \pi_{0}(z)\right| .
\end{aligned}
$$

We have shown $(A+\delta)\left|\operatorname{Im} \pi_{0}(z)\right| \leq A\left|\operatorname{Im} \pi_{0}(z)\right|$. This implies that $\pi_{0}(z)$ is real, hence also $z$ is real.

The same arguments apply to the opposite cone $\Gamma_{0}^{-}$.

Proof of Theorem [10. (This proof follows very closely the one of [5], Theorem 5.1.) Contrary to the convention in the remainder of the paper, we will work here with the norm $|z|:=\max _{j=1, \ldots, n}\left|z_{j}\right|$. We may assume $\xi=0$. Let $W_{1}, \ldots, W_{q}$ denote the irreducible components of $T_{0} V$. By hypothesis, there exist $\xi_{j} \in W_{j} \cap\left(\mathbb{R}^{n} \backslash\{0\}\right)$ so that $V$ is 1 -hyperbolic with respect to $\xi_{j}$ and to $-\xi_{j}, j=1, \ldots, q$. It follows from Proposition 12 that, if we perturb the $\xi_{j}$ a little if necessary, then there is a common noncharacteristic projection $\pi$ that works for all $\pm \xi_{j}, j=1, \ldots, q$. We assume it to be $\pi:\left(z_{1}, \ldots, z_{n}\right) \mapsto\left(z_{1}, \ldots, z_{k}\right)$ where $k:=\operatorname{dim} V$.

Let $D \in \mathbb{C}\left[z_{1}, \ldots, z_{k}\right]$, homogeneous of some degree $d$, be chosen so that $T_{0} V$ is unbranched over $\left\{w \in \mathbb{C}^{k} \mid D(w) \neq 0\right\}$. (See, e.g., Whitney [23], I.8E, or Chirka 
[10], 1.3.) For $0<\eta<1$ let

$$
S(\eta):=\left\{z \in \mathbb{C}^{n}|| D\left(z_{1}, \ldots, z_{k}\right)|\leq \eta| z_{1}, \ldots,\left.z_{k}\right|^{d}\right\} .
$$

Since it is possible to perturb the $\xi_{j}$ a little and still keep the same projection $\pi$, we may assume $D\left(\xi_{j}\right) \neq 0$ and hence $\xi_{j} \notin S(\eta)$ if $\eta$ is chosen sufficiently small. Pick $\delta>0$ so small that

$$
S(\eta) \cap \bigcup_{\substack{j=1 \\ \sigma= \pm 1}}^{q} \Gamma\left(\sigma \xi_{j}, B(0, \delta), \delta\right)=\emptyset
$$

and

$$
\text { if } z \in V \cap \Gamma\left( \pm \xi_{j}, B(0, \delta), \delta\right) \text { and } \pi(z) \text { is real, then } z \text { is real. }
$$

Since $T_{0} V$ is unbranched over $\pi(S(\eta))$, it is not difficult to see that for sufficiently small positive $\delta_{1}<\delta$ the projection $\pi$ induces an analytic map

$$
\tilde{\pi}: V \cap B\left(0, \delta_{1}\right) \backslash S(\eta) \rightarrow T_{0} V
$$

such that for $z \in V$ the point $\tilde{\pi}(z)$ is the one in $T_{0} V$ satisfying $\pi(z)=\pi \circ \tilde{\pi}(z)$ which is nearest to $z$. Note that if $\eta>0$ is small enough, then the sets $W_{j} \backslash S(\eta)$ are connected manifolds.

Fix such a number $\eta$ and set

$$
R(j):=\mathbb{C} \cdot\left(\Gamma\left(\xi_{j}, B(0, \delta), \delta\right) \cap W_{j} \cap \mathbb{R}^{n}\right) \quad \text { and } \quad \mathcal{R}:=\bigcup_{j=1}^{q} R(j) .
$$

Since each $W_{j} \backslash S(\eta)$ is connected, it is not difficult to see that $\mathcal{R}$ is nonpluripolar in $T_{0} V \cap B(0, r) \backslash S(\eta)$. Now let $u \in \operatorname{PSH}(V)$ satisfy

$$
u(z) \leq 1, z \in V, \quad \text { and } \quad u(z) \leq 0, z \in V \cap \mathbb{R}^{n} .
$$

In order to show that for some constant $A_{0}>0$, which does not depend on $u$, the estimate

$$
u(z) \leq A_{0}|z|, z \in V,
$$

holds, we will apply Theorem 11 with $W:=T_{0} V, \Gamma:=T_{0} V \backslash S(\eta)$, and $\Gamma^{\prime}:=$ $\mathbb{C}^{k} \backslash \pi(S(\eta))$ to suitable functions, derived from the given function $u$. To define them, fix a small number $a>0$ and define the function $u_{0}$ on $T_{0} V \cap B\left(0, \delta_{1}\right) \backslash S(\eta)$ by

$$
u_{0}(w):=\max \{u(z) \mid z \in V, \tilde{\pi}(z)=w\}
$$

and a function $u_{1}$ on $T_{0} V \backslash S(\eta)$ by

$$
u_{1}(w):= \begin{cases}\max \left(0, u_{0}(w)-a, 3+2\left(\log |w|-\log \delta_{1}\right)\right), & \text { if }|w|<\delta_{1}, \\ \left.3+2\left(\log |w|-\log \delta_{1}\right)\right), & \text { otherwise. }\end{cases}
$$

It follows from Hörmander [12], 4.4, that the singularities at the points where the maximum moves from one branch to another are removable, i.e., that $u_{0}$ is plurisubharmonic. This argument together with the fact that $u_{0}(w) \leq 1<3+$ $2\left(\log |w|-\log \delta_{1}\right)$ whenever $\delta_{1} / e<|w|<\delta_{1}$ also shows that $u_{1}$ is plurisubharmonic. 
We define $v_{0}$ on $B\left(0, \delta_{1}\right) \subset \mathbb{C}^{k}$ and $v_{1}$ on $\mathbb{C}^{k}$ by

$$
\begin{aligned}
& v_{0}(w):=\max (u(z) \mid z \in V, \pi(z)=w\}, \quad|w|<\delta_{1}, \\
& v_{1}(w):= \begin{cases}\max \left(0, v_{0}(w)-a, 3+2\left(\log |w|-\log \delta_{1}\right)\right), & \text { if }|w|<\delta_{1}, \\
\left.3+2\left(\log |w|-\log \delta_{1}\right)\right), & \text { otherwise. }\end{cases}
\end{aligned}
$$

The functions $v_{0}$ and $v_{1}$ are plurisubharmonic.

If $x \in W_{j} \cap \Gamma\left(\xi_{j}, B(0, \delta), \delta_{1}\right) \cap \mathbb{R}^{n}$ for some $j$ and $|x|>\delta_{1} / 2$, then $u_{0}(\zeta x) \leq 1$ for all $\zeta \in \mathbb{C}$ with $|\zeta| \leq 1$ and $u_{0}(\xi x) \leq 0$ for $-1 \leq \xi \leq 1$. By a standard potential theory argument (see, e.g., Nevanlinna [19], 38), there is a constant $C>0$, not depending on anything, such that $u_{0}(\zeta x) \leq C|\zeta|$ provided $|\zeta| \leq 1 / 2$. We have shown

$$
u_{0}(w) \leq \frac{2 C}{\delta_{1}}|w|, \quad w \in \mathcal{R} \cap B\left(0, \delta_{1} / 2\right) .
$$

Next define $\phi: T_{0} V \backslash S(\eta) \rightarrow[-\infty, \infty[$ by

$$
\phi(z):=\sup _{\zeta \in \mathbb{C}} \frac{u_{1}(\zeta z)}{|\zeta|}, \quad z \in T_{0} V \backslash S(\eta) .
$$

Then $\phi$ is positive homogeneous. Since $u_{1}$ is plurisubharmonic, the upper regularization $\phi^{*}$ is plurisubharmonic and positive homogeneous. Let $z \in \mathcal{R}$. If $z \in T_{0} V \cap B\left(0, \delta_{1}\right)$ satisfies $|z| \geq \delta_{1} / 2$, then $u_{0}(z) \leq 1 \leq\left(2 / \delta_{1}\right)|z|$. Since $3+2\left(\log |z|-\log \delta_{1}\right) \leq 2 \sqrt{e}|w|$, these considerations and (5) show

$$
\phi(z) \leq M|z| \text { for } z \in \mathcal{R} \quad \text { where } M:=\max \left(2 C / \delta_{1}, 2 \sqrt{e} / \delta_{1}\right) .
$$

Since each $z \in \mathcal{R}$ with $z \neq 0$ is a regular point of $T_{0} V$, the same estimate holds for $\phi^{*}$. Now define similarly to $\phi$ the function $\psi: \mathbb{C}^{k} \rightarrow[-\infty, \infty[$ by

$$
\psi(w):=\sup _{\zeta \in \mathbb{C}} \frac{v_{1}(\zeta w)}{|\zeta|}, \quad w \in \mathbb{C}^{k} .
$$

$\psi$ is an extension of $w \mapsto \max \left\{\phi(z) \mid z \in T_{0} V \backslash S(\eta), \pi(z)=w\right\}$ and consequently $\psi^{*}$ extends $w \mapsto \max \left\{\phi^{*}(z) \mid z \in T_{0} V \backslash S(\eta), \pi(z)=w\right\}$. Therefore we can apply Theorem 11 with $W=T_{0} V, \Gamma=T_{0} V \backslash S(\eta), \Gamma^{\prime}=\mathbb{C}^{k} \backslash \pi(S(\eta)), u=\phi^{*}$, and $v=\psi^{*}$. It gives the existence of $A \geq 1$ such that

$$
\psi^{*}(w) \leq A|w| \text { for all } w \in \mathbb{C}^{k} .
$$

If $\psi^{*}$ is expressed in terms of $u$, this estimate implies $\mathrm{RPL}_{\text {loc }}(0)$ if $a$ tends to 0 .

Corollary 13. Let $f$ be a holomorphic function, defined on the ball $B(0, r) \subset \mathbb{C}^{n}$ for some $r>0$, which satisfies the following conditions:

(a) $f$ is real on $B(0, r) \cap \mathbb{R}^{n}$,

(b) the localization $f_{0}$ of $f$ at zero is squarefree and has positive degree,

(c) for each irreducible factor $q$ of $f_{0}$, the real zero set of $q$ has dimension $n-1$ at zero.

Then $V=\{z \in B(0, r): f(z)=0\}$ satisfies $\operatorname{RPL}_{\mathrm{loc}}(0)$.

Proof. The hypotheses imply that $f$ can be decomposed as $f=f_{0}+g$, where $f_{0}$ is a homogeneous polynomial of degree $m>0$ and where $g$ is holomorphic on $B(0, r)$ and satisfies for some constant $C>0$ the estimate

$$
|g(z)| \leq C|z|^{m+1}, \quad z \in B(0, r / 2) .
$$


Note that $f_{0}$ has real coefficients. Then the hypotheses imply $f_{0}=\prod_{j=1}^{s} P_{j}$, where $P_{1}, \ldots, P_{s}$ are irreducible homogeneous polynomials with real coefficients which are pairwise not proportional. By Whitney [23], 7.4D (see Definition 5), we have

$$
T_{0} V=\left\{z \in \mathbb{C}^{n}: f_{0}(z)=0\right\}=\bigcup_{j=1}^{s} W_{j}
$$

where the varieties

$$
W_{j}:=\left\{z \in \mathbb{C}^{n}: P_{j}(z)=0\right\}, \quad 1 \leq j \leq s,
$$

are the irreducible components of $T_{0} V$.

Next note that the hypotheses on $f_{0}$ imply that for $1 \leq j \leq s$ we can choose $\xi_{j} \in W \cap B(0, r / 2) \cap \mathbb{R}^{n}$ such that $\operatorname{grad} P_{j}\left(\xi_{j}\right) \neq 0$ and $\xi_{j} \notin W_{k}$ for $k \neq j$. Then we have

$$
\operatorname{grad} f_{0}\left(\xi_{j}\right)=\left(\prod_{\substack{k=1 \\ k \neq j}}^{s} P_{k}\left(\xi_{j}\right)\right) \operatorname{grad} P_{j}\left(\xi_{j}\right) \neq 0, \quad 1 \leq j \leq s .
$$

For fixed $j$, we may assume that $\frac{\partial f_{0}}{\partial z_{n}}\left(\xi_{j}\right) \neq 0$ and write $z=\left(z^{\prime}, z_{n}\right)$ for $z \in \mathbb{C}^{n}$. Then an application of the real and the complex implicit function theorem implies that for suitable numbers $\delta_{1}, \delta_{2}>0$ the variety $W_{j}$ in a neighborhood of $\xi_{j}$ is the graph of a holomorphic function $h_{j}: B\left(\xi_{j}^{\prime}, \delta_{1}\right) \rightarrow B\left(\xi_{j, n}, \delta_{2}\right)$ which is real over $B\left(\xi_{j}^{\prime}, \delta_{1}\right) \cap \mathbb{R}^{n-1}$. Of course, we may choose $\delta_{1}$ and $\delta_{2}$ so small such that $\frac{\partial f_{0}}{\partial z_{n}}(z) \neq 0$ for $z \in B\left(\xi_{j}^{\prime}, \delta\right) \times B\left(\xi_{j, n}, \delta\right)$, where $\delta:=\min \left(\delta_{1}, \delta_{2}\right)$. Since $\xi_{j, n}$ is a simple zero of $\lambda \mapsto f_{0}\left(\xi_{j}^{\prime}, \lambda\right)$, we can choose $0<\rho<\delta / 4$ so that $\left|f_{0}\left(\xi_{j}^{\prime}, \lambda\right)\right|>0$ for $0<\left|\lambda-\xi_{j, n}\right| \leq$ $\rho$. Thus

$$
a:=\inf \left\{\left|f_{0}\left(\xi_{j}^{\prime}, z_{n}\right)\right|:\left|z_{n}-\xi_{j, n}\right|=\rho\right\}>0 .
$$

Hence we can choose $0<\delta_{3}<\delta / 2$ such that

$$
\inf \left\{\left|f_{0}\left(\zeta^{\prime}, \lambda\right)\right|:\left|\zeta^{\prime}-\xi_{j}^{\prime}\right| \leq \delta_{3},\left|z_{n}-\xi_{j, n}\right|=\rho\right\} \geq \frac{a}{2} .
$$

Now note that for $0<t \leq 1,\left|\zeta^{\prime}-\xi_{j}^{\prime}\right| \leq \delta_{3}$, and $\left|z_{n}-\xi_{j, n}\right|=\rho$, the estimate (6) implies

$$
\left|g\left(t \zeta^{\prime}, t z_{n}\right)\right| \leq C t^{m+1}\left|\left(\zeta^{\prime}, z_{n}\right)\right|^{m+1} \leq C\left(\left|\xi_{j}^{\prime}\right|+\left|\xi_{j, n}\right|+\delta\right)^{m+1} t^{m+1},
$$

while

$$
\left|f_{0}\left(t \zeta^{\prime}, t z_{n}\right)\right|=t^{m}\left|f_{0}\left(\zeta^{\prime}, z_{n}\right)\right| \geq \frac{a}{2} t^{m}
$$

These estimates show the existence of $0<t_{0} \leq 1$ such that for each $0<t \leq t_{0}$ and each $\zeta^{\prime} \in \mathbb{C}^{n-1}$ with $\left|\zeta^{\prime}-\xi_{j}^{\prime}\right|<\delta_{3}$ we can apply Rouché's Theorem to see that the equation $f\left(t \zeta^{\prime}, z_{n}\right)=0$ has exactly one solution $\zeta_{n} \in \mathbb{C}$ satisfying $\left|\zeta_{n}-t \xi_{j}\right|<$ $t \rho$. Since $f$ is real for real arguments and the neighborhood in which the root is unique is symmetric with respect to complex conjugation, it follows that $V$ is 1 hyperbolic with respect to $\xi_{j}$. Since the same arguments apply to $-\xi_{j}$, the proof is complete. 
Example 14. The sufficient condition in Theorem 10 is not necessary. In fact, for

$$
P\left(s, w_{1}, w_{2}\right):=\left(s^{2}-w_{1}^{2}\right)^{2}-w_{2}\left(w_{1}^{2}-w_{2}^{2}\right)\left(w_{1}^{2}+w_{2}^{2}\right)
$$

the variety $V(P)$ satisfies $\mathrm{RPL}_{\mathrm{loc}}(0)$, although the hypotheses of Theorem 10 are violated. This example is an adaptation of Example 4.10 of Bainbridge [2]. For the proof we refer to [7].

\section{REFERENCES}

[1] Andreotti, A., Nacinovich, M., Analytic convexity and the principle of Phragmén-Lindelöf, Quaderni della Scuola Normale de Pisa (1980). MR 83h:32018

[2] Bainbridge, D., Phragmén-Lindelöf estimates for plurisubharmonic functions of linear growth, Thesis, Ann Arbor 1998.

[3] Boiti, C., Nacinovich, M., The overdetermined Cauchy problem, Ann. Inst. Fourier (Grenoble) 47 (1997), 155-199. MR 98a:35095

[4] Braun, R.W., Hörmander's Phragmén-Lindelöf principle and irreducible singularities of codimension 1, Boll. Un. Mat. Ital. 6 - A (1992), 339-348. MR 94b:35012

[5] Braun, R.W., Meise, R., Taylor, B.A., A radial Phragmén-Lindelöf estimate for plurisubharmonic functions on algebraic varieties, Ann. Polon. Math. LXXII (1999), 159-179. MR 2001b:32068

[6] Braun, R.W., Meise, R., Taylor, B.A., Algebraic varieties on which the classical PhragménLindelöf estimates hold for plurisubharmonic functions, Math. Z. 232 (1999), 103-135. MR 2001d:32048

[7] Braun, R.W., Meise, R., Taylor, B.A., An example concerning the local radial PhragménLindelöf condition, to appear in Recent Progress in Functional Analysis, Proceedings of the International Functional Analysis Meeting on the Occasion of the 70th Birthday of Professor Manuel Valdivia, K. D. Bierstedt, J. Bonet, M. Maestre, J. Schmets (Eds.), North-Holland Math. Studies.

[8] Braun, R.W., Meise, R., Taylor, B.A., The geometry of analytic varieties satisfying the local Phragmén-Lindelöf condition and a geometric characterization of partial differential operators that are surjective on $\mathcal{A}\left(\mathbb{R}^{4}\right)$, manuscript.

[9] Braun, R.W., Meise, R., Vogt, D., Characterization of the linear partial differential operators with constant coefficients which are surjective on non-quasianalytic classes of Roumieu type on $\mathbb{R}^{N}$, Math. Nachr. 168 (1994), 19-54. MR 95g:35004

[10] Chirka, E. M., Complex Analytic Sets, Kluwer, Dordrecht, 1989. MR 92b:32016

[11] Franken, U., Meise, R., Extension and lacunas of solutions of linear partial differential equations, Ann. Inst. Fourier (Grenoble) 46 (1996), 154-161. MR 97h:35005

[12] Hörmander, L., On the existence of real analytic solutions of partial differential equations with constant coefficients, Invent. Math. 21 (1973), 151-183. MR 49:817

[13] Kaneko, A., On Hartogs type continuation theorem for regular solutions of linear differential equations with constant coefficients, J. Fac. Sci. Univ. Tokyo Sect. IA 35 (1988), 1-26. MR 89m:35043

[14] Meise, R., Taylor, B.A., Phragmén-Lindelöf conditions for graph varieties, Result. Math. 36 (1999), 121-148. MR 2000j:32057

[15] Meise, R., Taylor, B.A., Vogt, D., Characterization of the linear partial differential operators with constant coefficients that admit a continuous linear right inverse, Ann. Inst. Fourier (Grenoble) 40 (1990), 619-655. MR 92e:46083

[16] Meise, R., Taylor, B.A., Vogt, D., Extremal plurisubharmonic functions of linear growth on algebraic varieties, Math. Z. 219 (1995), 515-537. MR 96j:32019

[17] Meise, R., Taylor, B.A., Vogt, D., Phragmén-Lindelöf principles on algebraic varieties, J. Amer. Math. Soc. 11 (1998), 1-39. MR 98j:32007

[18] Momm, S., On the dependence of analytic solutions of partial differential equations on the right hand side, Trans. Amer. Math. Soc. 345 (1994), 729-752. MR 95a:46036

[19] Nevanlinna, R., Eindeutige analytische Funktionen, Springer, Berlin, Heidelberg, New York 1974. MR 49:9165 
[20] Palamodov, V.I., A criterion for splitness of differential complexes with constant coefficients, in Geometrical and Algebraical Aspects in Several Complex Variables, C.A. Berenstein and D.C. Struppa (Eds.), Edit El (1991), pp. 265-290. MR 94d:58137

[21] Sibony, N., Wong, P., Some results on global analytic sets, Séminaire Lelong-Skoda (Analyse), Springer Lecture Notes, Vol. 822 (1978-79), 221-237. MR 82h:32017

[22] Siciak, J., Extremal plurisubharmonic functions and capacities in $\mathbb{C}^{n}$, Sophia Kokyuroku in Mathematics 14, Tokyo 1982.

[23] Whitney, H., Complex Analytic Varieties. Addison-Wesley, Reading, Mass., 1972. MR 52:8473

[24] Zampieri, G., An application of the fundamental principle of Ehrenpreis to the existence of global solutions of linear partial differential equations, Boll. Un. Mat. Ital. 6 (1986), 361-392. MR 82m:35018b

Mathematisches Institut, Heinrich-Heine-Universität, Universitätsstrasse 1, 40225 DÜSSEldoRF, GERMANY

E-mail address: Ruediger.Braun@uni-duesseldorf.de

Mathematisches Institut, Heinrich-Heine-Universität, Universitätsstrasse 1, 40225 DÜSSELDORF, GERMANY

E-mail address: meise@cs.uni-duesseldorf.de

Department of Mathematics, University of Michigan, Ann Arbor, Michigan 48109

E-mail address: taylor@umich.edu 